\title{
Meta-analysis of Chicken - Salmonella infection experiments
}

\author{
Marinus FW te Pas ${ }^{1 *}$, Ina Hulsegge ${ }^{1}$, Dirkjan Schokker ${ }^{1}$, Mari A Smits ${ }^{1,2}$, Mark Fife ${ }^{3}$, Rima Zoorob ${ }^{4}$, \\ Marie-Laure Endale ${ }^{5}$ and Johanna MJ Rebel ${ }^{2}$
}

\begin{abstract}
Background: Chicken meat and eggs can be a source of human zoonotic pathogens, especially Salmonella species. These food items contain a potential hazard for humans. Chickens lines differ in susceptibility for Salmonella and can harbor Salmonella pathogens without showing clinical signs of illness. Many investigations including genomic studies have examined the mechanisms how chickens react to infection. Apart from the innate immune response, many physiological mechanisms and pathways are reported to be involved in the chicken host response to Salmonella infection. The objective of this study was to perform a meta-analysis of diverse experiments to identify general and host specific mechanisms to the Salmonella challenge.

Results: Diverse chicken lines differing in susceptibility to Salmonella infection were challenged with different Salmonella serovars at several time points. Various tissues were sampled at different time points post-infection, and resulting host transcriptional differences investigated using different microarray platforms. The meta-analysis was performed with the R-package metaMA to create lists of differentially regulated genes. These gene lists showed many similarities for different chicken breeds and tissues, and also for different Salmonella serovars measured at different times post infection. Functional biological analysis of these differentially expressed gene lists revealed several common mechanisms for the chicken host response to Salmonella infection. The meta-analysis-specific genes (i.e. genes found differentially expressed only in the meta-analysis) confirmed and expanded the biological functional mechanisms.

Conclusions: The meta-analysis combination of heterogeneous expression profiling data provided useful insights into the common metabolic pathways and functions of different chicken lines infected with different Salmonella serovars.
\end{abstract}

\section{Background}

Chicken meat and eggs for human consumption can be contaminated with several Salmonella species, and therefore chicken-derived food products can be regarded as a source of human zoonotic pathogens. Although proper food preparation should kill the pathogens, the food items contain a potential hazard for humans. In chicken both acute fatal and chronic Salmonellosis occurs depending upon the infecting Salmonella serovar [1-4]. Broad host range Salmonella serovars used most

\footnotetext{
* Correspondence: marinus.tepas@wur.nl

'Animal Breeding and Genetics Centre (ABGC), Wageningen UR Livestock Research, Animal Sciences Group, Wageningen University and Research Centre, P.O. Box 65, 8200 AB Lelystad, The Netherlands

Full list of author information is available at the end of the article
}

often in studies - including the studies used for this meta-analysis, S. Typhimurium and $S$. Enteritidis, do not cause fatal infections when chickens older than one day post hatch are orally challenged. Chickens can harbor Salmonella pathogen without showing clinical signs of illness [3,5]. Many investigations have examined the mechanisms how chickens react to infection, the mechanism of transfer to humans and host immunity to infection $[3,6]$.

Diverse host species may react differently to Salmonella infection [7]. While one-day old chickens may succumb to broad host range Salmonella infection, older chickens often show no clinical signs. Furthermore, specific chicken lines have been shown to differ in their susceptibility for Salmonella [8-11]. These clear genetic differences in susceptibility may be due to pleiotropic effects, or to

\section{Biomed Central}


unknown selection-related mechanisms. In the last decade, gene expression profiling studies using microarrays have been widespread in animal genomics and have enabled researchers to monitor the effects of pathogens on host cells and tissues with the aim of gaining insights into the molecular mechanisms that are involved in the host-pathogen interactions. Several genes involved in Salmonella susceptibility in chicken have been determined [12-17]. Apart from the innate immune response, many physiological mechanisms and pathways were reported to be involved in the chicken host response to Salmonella infection which are also active in uninfected cells, including energy metabolism, cell shape, and others [18-20].

Each of these independent experiments showed how individual hosts within the specific experimental conditions reacted to Salmonella infection. Meta-analysis of these experiment may reveal a common genetic background for the chicken host reaction to the Salmonella infection. Furthermore, the age-related differences in the mechanisms and the outcome of the host immuneresponse to Salmonella infection suggests that different immune-reactions are possible, and are likely to be age related $[21,22]$. Taken together this indicates a complex interplay between chicken host genetics and Salmonella serovars $[3,10,11,18,23]$.

Meta-analysis methods integrate results of independent studies creating very large datasets with increased statistical power [24,25]. It allows a more objective appraisal of evidence than individual studies, and has been widely used to interpret contradictory results from diverse studies. Furthermore, this analysis method overcomes the problem of reduced statistical power associated with studies of small sample size (reviewed by $[26,27]$. Such methods enable analyses at a higher level than possible on the individual datasets. Host-specific general mechanisms can be determined in addition to mechanisms operating under specific conditions. Thus, using previously published individual datasets we were able to highlight new results that contribute to understanding of common disease mechanisms and physiology. Different experiments were performed under the umbrella of a large EU-funded project called SABRE Cutting Edge Genomics for Sustainable Animal Breeding $[9,21,22,28,29]$. This meta-analysis brings the individual studies together offering the potential to highlight new host-pathogen interaction mechanisms and elucidate possible general host-response mechanisms. The objective of this study was to determine the general chicken host response to Salmonella infection independent of age of the chicken, age at infection and, time post infection and independent of host response time post-infection. The results indicate several common chicken host reaction mechanisms to Salmonella infection.

\section{Methods}

\section{Animals and Salmonella challenges}

Experiment 1

The original animal experiment was described by Fife et al. [29]. In short, two inbred chicken lines differing in susceptibility to gut pathogens (lines $\mathrm{N}$ and 6, with line 6 more resistant than line $\mathrm{N}$, [29]) were at three weeks of age orally infected with $5.1 \times 10^{7}-1.97 \times 10^{8}$ cfu $S$. Typhimurium according to the method of Barrow et al. [30]. The caecal tonsils and spleens were sampled at 2, 3, and 4 days post infection, $(\mathrm{n}=10)$, and four birds at each time point were used as uninfected controls. Total RNA for these samples was isolated and used for hybridization to the $20.6 \mathrm{~K}$ chicken oligo array (ARK genomics; http:// www.ark-genomics.org/) microarrays. Infection and infection clearance was determined by ceacal counts of $S$. Typhimurium ( $c f u 10^{6} \mathrm{l}$ ) and differences between the lines investigated. A total of 32 microarrays per line were obtained.

\section{Experiment 2}

The original experiment was described by Schokker et al. [31] (GEO data: GSE27069). In short, three commercial chicken lines differing for Salmonella sensitivity were orally infected with $10^{5} \mathrm{cfu} S$. Enteritidis at the day of hatch. The jejunum was sampled at $8 \mathrm{~h}$ and days 1 and 2 post infection, 10 animals each, of which 5 were used for microarray analysis. A reference pool was created from $0.33,1$ and 2 days post infection birds, for all three lines together, as well as control and infected birds. Infection was checked by body weight and liver weight gain and liver clearance, and cloaca swaps. Total RNA was isolated and hybridized to the same microarrays as experiment 1 . A total of 45 microarrays were obtained [31].

\section{Experiment 3}

The original experiment was described by Schokker et al. $[16,28]$ (ArrayExpress data: E-MEXP-042). In short, chickens were challenged orally at the day of hatch with $10^{5} \mathrm{cfu} S$. Enteritidis. The jejunum was sampled at $8 \mathrm{~h}$, and $1,2,4,8,12$, and 21 days post infection, 5 animals each for both control and infected situation. Infection was checked by body weight and liver weight gain and liver clearance. Total RNA was isolated and single color hybridized against Agilent chicken microarrays. A total of 70 microarrays were obtained $[16,21]$.

\section{Experiment 4}

The original experiment was described by van Hemert et al. [21] (GEO data: GSE3702). In short, two chicken lines differing in growth rate and Salmonella sensitivity were orally infected with $10^{5}$ cfu of $S$. Enteritidis at one day of age and jejunum samples were taken after $24 \mathrm{~h}$. A 
non-infected control was used, 5 chicken each. Total RNA was isolated and hybridized against Affymetrix chicken microarrays using group comparison. A total of four microarrays were obtained [21].

\section{Meta-analysis methodology Pre-processing microarray data}

The microarray data pre-processing was carried out using functions from the LIMMA package (version 3.2.1) [32]. The quality of the arrays was evaluated through several diagnostic plots. The "normexp" method [33] was used for background correction, followed by normalization within individual microarrays using the default "print tip loess" method and normalization between arrays using the "quantile" method. The background correction was set to: offset $=50$. The offset can be used to add a constant to the intensities before logtransforming, so that the log-ratios are shrunk towards zero at the lower intensities. This may eliminate or reverse the usual 'fanning' of log-ratios at low intensities associated with local background subtraction. Areas with higher than average background were removed from the results. Bad hybridization always removed whole microarrays. Especially in experiment 1 this removed parts of the results. After normalization 20 slides of experiment 1 and one slide of Experiment 2 were deleted due to poor quality hybridization. This will inevitably affect the results, but this procedure ensures that only good quality data were used.

\section{Meta-analysis}

Meta-analysis was carried out using the directpvalcombi function from the metaMA package (Meta-analysis for MicroArrays) (version 1.1) in $\mathrm{R}$ [24]. The input for the meta-analysis were the individual microarrays of all experiments. The meta-analysis produced lists of gene names with differential expression under specific conditions. The lists of genes were grouped in (1) DE: the list of Differentially Expressed genes (i.e. in the experiments and in the meta-analysis), and (2) IDD (Integration Driven Discoveries): the list of genes that were determined differentially expressed in the meta-analysis that were not identified in any of the individual studies alone (i.e. new differentially expressed genes). For both DE and IDD gene lists (e) experiment (i.e. 4 studies) and (t) time (14 studies) were generated. Subsequently from these (e) and $(\mathrm{t})$ the following groups were also extracted, namely (et): overlap between the (e) and $(\mathrm{t})$ groups, $(\mathrm{e}-\mathrm{t})$ : genes unique in (e), and (t-e): genes unique in (t) (Figure 1). The groups included results from the different tissues. Since the analyses focus on expression differences related to Salmonella infection no interaction with tissue-specific gene expression can be expected.

\section{Functional bioinformatics analyses}

The lists of differentially expressed genes were analyzed for biological functionalities using the DAVID (The Database for Annotation, Visualization and Integrated Discovery) software [34-36], version 6.6. The gene lists were analyzed against the gene list of the human genome since the annotation of the human genome, especially with physiological data, is more advanced than the chicken genome. Therefore, all genes were converted to human Entrez identifiers. The false discovery rate, multiple testing correction for statistical significance [37], and the fold enrichment analyses were manually included for all analyses. The tissue-specific profiles and functional annotations and clusterings of the gene lists were investigated.

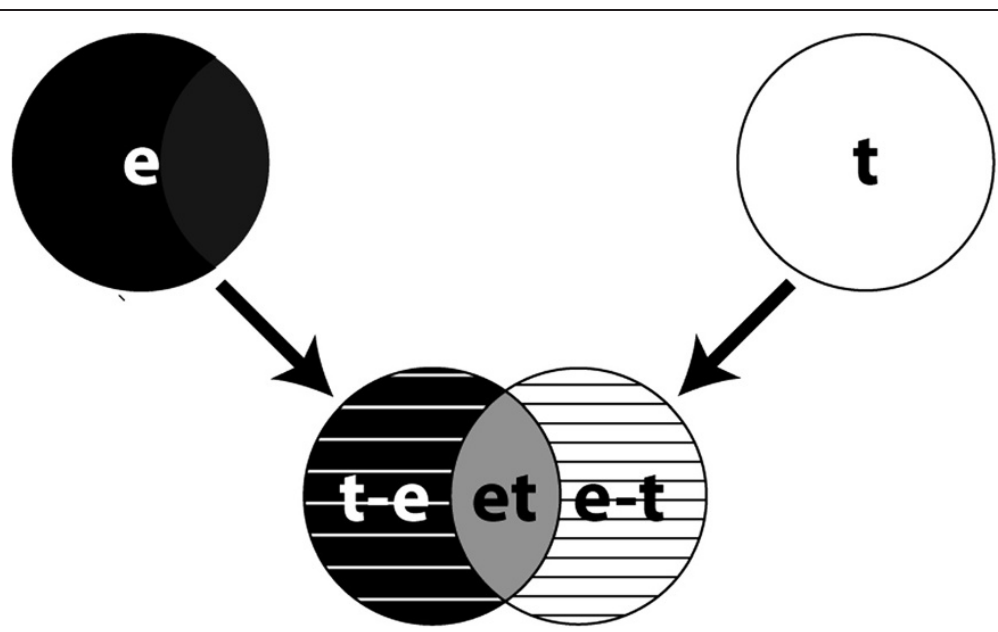

Figure 1 Overview experiment and time analyses. For both IDD and DE experiment $(\mathrm{e})$ and time (t) gene lists were generated. Thereafter different subsets were extracted, unique time genes (t-e), unique experiment genes (e-t), and overlap between (e) and (t) (et). 


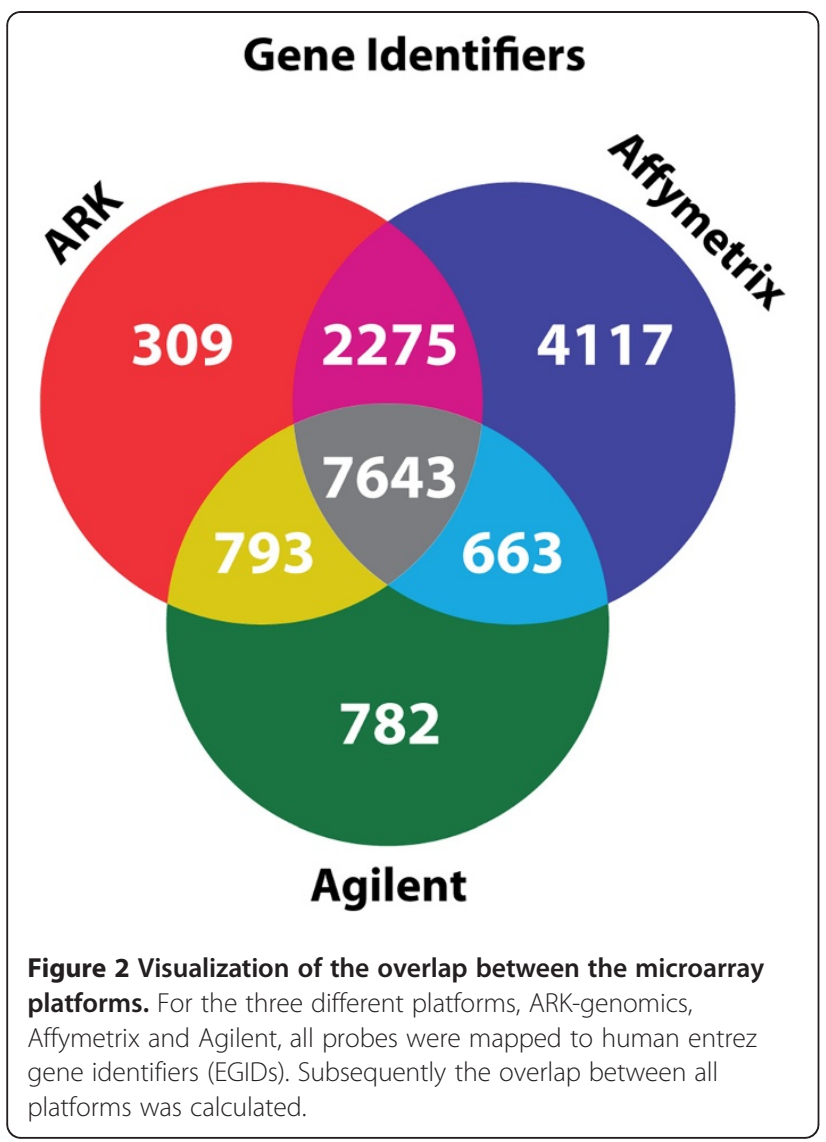

\section{Results}

\section{Meta-analysis}

Due to the platform differences used in the individual studies the number of genes available in all studies was reduced as expressed in Figure 2. The Figure shows that 7,643 genes were common to all microarray platforms and thus available for meta-analysis over all studies. Different platforms may use different probes for the same genes, and the probes may differ in hybridization characteristics. However, since differential expression of genes was measured within a platform for each of the experiments this will not affect the meta-analysis.

The results of the meta-analysis DE-group were expressed in several lists of genes with regulated expression in more than one or all experiments (Table 1 - the Table with the gene lists is Additional file 1). The Table shows that approximately 3,000 genes are differently expressed, irrespective of age of infection or Salmonella serovar of infection. Differential expression was between control and infected animals of the same age. Furthermore, most differently expressed genes were the same in all experiments irrespective of sampling time point post-infection (Figure 3). The Figure shows that the large majority of the genes are shared by the (e) and the (t) categories.
Table 1 Number of differentially expressed genes per group

\begin{tabular}{lll}
\hline Category & DE & IDD \\
\hline e & 2942 & 85 \\
$\mathbf{t}$ & 3227 & 378 \\
et & 2861 & 61 \\
e-t & 81 & 24 \\
t-e & 366 & 317 \\
\hline
\end{tabular}

Number of differentially expressed genes in the groups Differentially Expressed (DE) genes and Integration Driven Discoveries (IDD), the latter of which is specific for genes only found in the meta-analysis. Categories depend on the experimental differences: (e) experiment, (t) time post infection, (et) both (e) and $(\mathrm{t}),(\mathrm{e}-\mathrm{t})$ experiment specific, and $(\mathrm{t}-\mathrm{e})$ time specific.

The IDD group (i.e. the list of genes found only differently expressed in the meta-analysis) genes were predominantly in the $(\mathrm{t})$ category (Figure 4). A few genes were differently expressed in the meta-analysis only for the (e) category.

\section{Functional bioinformatics analyses}

The gene lists were analyzed for biological functional groups using the DAVID software. First the expression

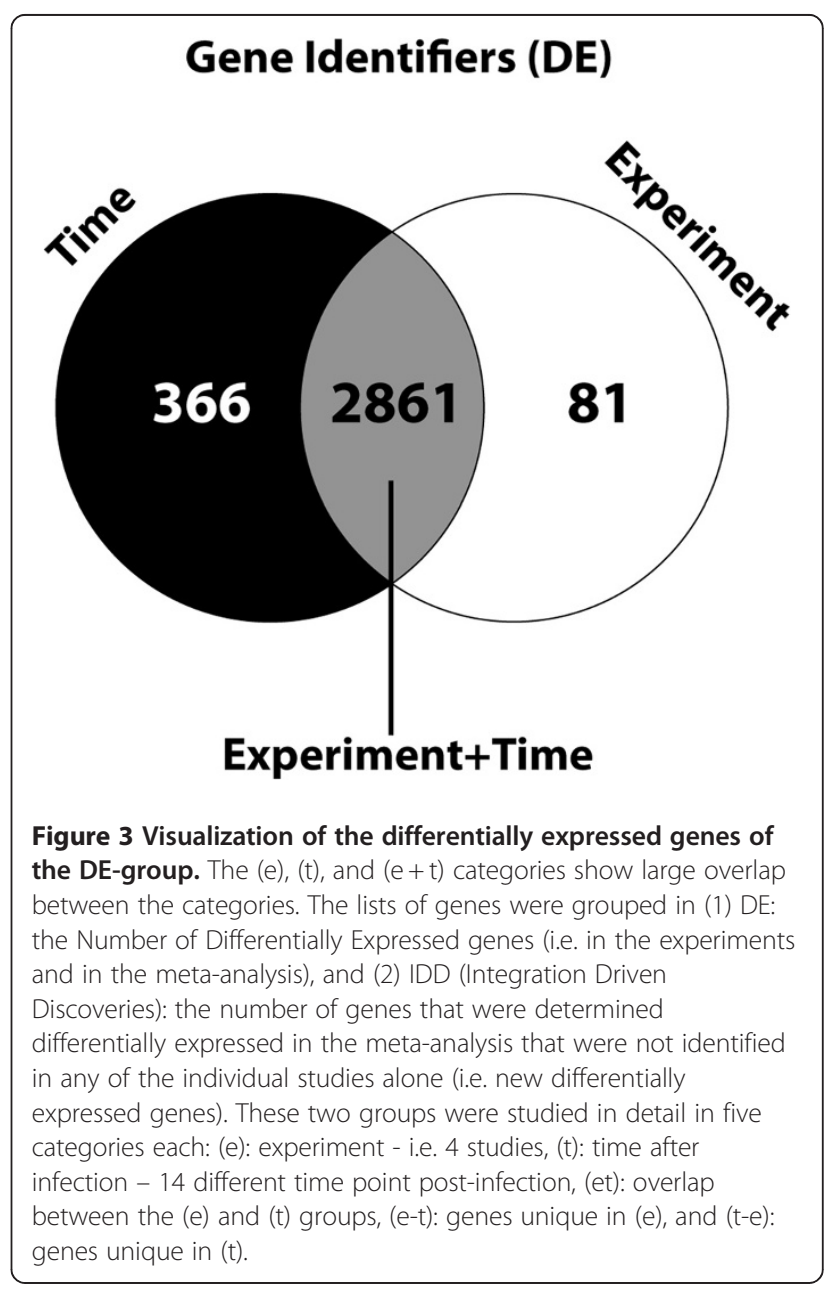




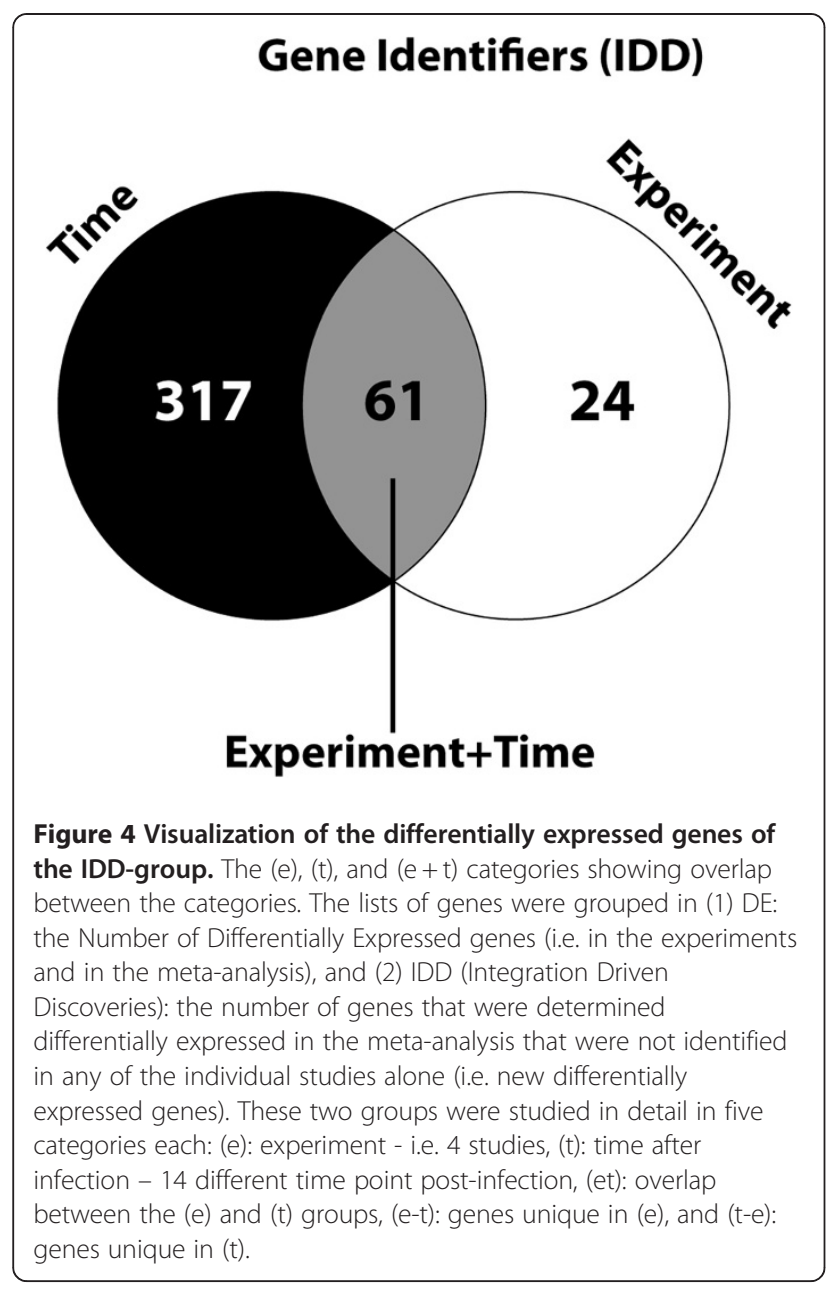

profile of differentially expressed genes of the DE-group was compared with normal tissue-specific expression profiles from the same time points and the same tissue type (Table 2). Significant results were only obtained for the DE group, (e), (t), and (et) categories. The Table indicates that the sampled intestinal tissue showed expression profiles related to a number of different tissue types. The most significant tissue expression profile is epithelium. This cell type is abundantly present in intestinal tissue. Several other cell types and tissues also showed similarities for tissue-specific expression profiles, some of them not relevant in intestine (data not shown).

The results of the differently expressed gene lists were then analyzed for functional biological mechanisms. The results are shown in Additional file 2. The results showed lists of biological functions for the (e) and the (t) categories of the DE group. Moreover, the (et) category showed that both lists were largely similar, and the top of the lists were even identical. The top of the lists indicated that phosphorylation of proteins, acetylation in the cytoplasm and lumen of other cellular components, and ATP consuming processes were important biological mechanisms during chicken host reaction to Salmonella infection. The meta-analysis showed additional significant results for both the experiment (e-t) and the time ( $t$ e) categories of the phosphoprotein biological function during chicken host reaction to Salmonella infection. Similarly, in the IDD group the time (t-e) category further indicated additional significant results especially for the phosphoprotein biological function.

Finally, a cluster analysis was performed for the lists of biological functional annotations. The DE-group (e) and (et) categories showed over 600 clusters. Due to the fact that the list of the $(\mathrm{t})$ category in the $\mathrm{DE}$ group was longer than 3000 entries, clustering was technically not possible for the DAVID software. Since the lists of functional annotations of the (et) and the ( $t$ ) categories of the DE group were very similar the $(\mathrm{t})$ category clusters were deduced from the (e) and (et) categories clusters (see below).

Table 3 shows the results for clusters with enrichment scores larger than 2. An enrichment score indicate whether the number of genes in a cluster is equal to the expected number of genes (due to the number of genes of that physiological group in the genome and on the microarray) or higher or lower than expected. A high enrichment score thus indicates that the physiological trait of the cluster may be significant to the trait. The clusters were ordered by enrichment scores. The range of enrichment scores was from over 10 to almost zero. Enrichment scores less than 2 were omitted, leaving between 35 and 40 clusters in the DE-group (e) and (et) categories, respectively. For completeness, the Additional file 3 includes the biological functions per cluster in detail, i.e. larger than 1 . The content of each cluster is a group of biological functions taken together from various databases centered on a specific theme. For example (see Table 3), cluster 1 groups biological functions together related to the lumen of cell organelles, especially relating to the nucleus. Cluster 2 groups nucleotide binding and phosphorylation functions (especially serine and threonine phosphorylation), while cluster 3 groups mitochondrial membrane functionalities. The Table contains both cell / tissue morphological clusters and (macro) molecular biogenesis functional clusters. The functions of several protein domains were also relevantly clustered.

Taken together functional clustering analysis showed that these functional annotations can be grouped together in higher order biological morphological structures and biological processes. The clusters can be divided in 21 clusters describing metabolic processes of which three were related to energy metabolism, eight clusters describing (cell) morphological features, three clusters specifically pointing to protein domains involved, one cluster related to apoptosis and one cluster 
Table 2 Tissue specificity of differentially expressed gene profiles

\begin{tabular}{|c|c|c|c|c|c|c|c|c|c|c|c|c|}
\hline \multirow[b]{2}{*}{ Tissue } & \multicolumn{3}{|c|}{ Experiment (e) } & \multicolumn{3}{|c|}{ (et) } & \multirow[b]{2}{*}{ Benjamini } & \multirow[b]{2}{*}{ FDR } & \multicolumn{2}{|c|}{ Time $(t)$} & \multirow[b]{2}{*}{ Benjamini } & \multirow[b]{2}{*}{ FDR } \\
\hline & $\mathrm{N}$ & $\mathrm{FE}$ & Benjamini & FDR & $\mathrm{N}$ & $\mathrm{FE}$ & & & & $\mathrm{FE}$ & & \\
\hline Epithelium & 636 & 1.56 & $1.50 \mathrm{E}-34$ & 4.99E-34 & 616 & 1.56 & $1.36 \mathrm{E}-32$ & $4.54 \mathrm{E}-32$ & 682 & 1.53 & $1.20 \mathrm{E}-34$ & $3.92 \mathrm{E}-34$ \\
\hline Liver & 476 & 1.48 & $2.68 \mathrm{E}-19$ & $1.79 \mathrm{E}-18$ & 464 & 1.48 & 7.21E-19 & $4.82 \mathrm{E}-18$ & 516 & 1.46 & $2.04 \mathrm{E}-20$ & 1.33E-19 \\
\hline Brain & 1443 & 1.17 & 4.35E-16 & 4.36E-15 & 1400 & 1.17 & 1.55E-14 & $1.55 \mathrm{E}-13$ & 1566 & 1.16 & $5.17 \mathrm{E}-16$ & 5.07E-15 \\
\hline Skin & 360 & 1.28 & $6.81 \mathrm{E}-06$ & $1.59 \mathrm{E}-04$ & 351 & 1.28 & $8.22 \mathrm{E}-06$ & $1.92 \mathrm{E}-04$ & 392 & 1.28 & $2.63 \mathrm{E}-06$ & $6.02 \mathrm{E}-05$ \\
\hline Lymph & 152 & 1.46 & $3.10 \mathrm{E}-05$ & 8.28E-04 & 148 & 1.47 & 4.44E-05 & 0.001 & 157 & 1.38 & 3.98E-04 & 0.016 \\
\hline Bone marrow & 160 & 1.41 & $1.76 \mathrm{E}-04$ & 0.005 & 158 & 1.43 & $8.78 \mathrm{E}-05$ & 0.003 & 178 & 1.43 & $1.45 \mathrm{E}-05$ & 0.000 \\
\hline Muscle & 170 & 1.34 & 0.001 & 0.049 & 167 & 1.35 & $9.43 \mathrm{E}-04$ & 0.035 & 189 & 1.36 & $1.46 \mathrm{E}-04$ & 0.005 \\
\hline Cajal-Retzius cell & 53 & 1.75 & 0.001 & 0.056 & 52 & 1.77 & 0.001 & 0.055 & 56 & 1.69 & 0.002 & 0.079 \\
\hline Skeletal muscle & 123 & 1.38 & 0.003 & 0.170 & 122 & 1.41 & 0.002 & 0.079 & 141 & 1.45 & 0.000 & 0.004 \\
\hline Colon & 229 & 1.25 & 0.003 & 0.179 & 224 & 1.26 & 0.003 & 0.157 & 238 & 1.19 & 0.035 & 2.520 \\
\hline Fetal brain cortex & 55 & 1.65 & 0.004 & 0.208 & 54 & 1.67 & 0.004 & 0.196 & 59 & 1.62 & 0.004 & 0.181 \\
\hline Heart & 119 & 1.34 & 0.013 & 0.791 & 112 & 1.29 & 0.043 & 3.784 & 130 & 1.34 & 0.008 & 0.433 \\
\hline Renal cell carcinoma & 21 & 2.17 & 0.017 & 1.111 & 21 & 2.23 & 0.013 & 0.777 & 21 & 1.99 & 0.042 & 3.417 \\
\hline Lung & 453 & 1.13 & 0.030 & 2.239 & 447 & 1.15 & 0.013 & 0.811 & 504 & 1.15 & 0.005 & 0.249 \\
\hline Hepatoma & 54 & 1.49 & 0.038 & 2.953 & 54 & 1.54 & 0.023 & 1.605 & 59 & 1.49 & 0.026 & 1.717 \\
\hline Embryonal rhabdomyosarcoma & 9 & 3.34 & 0.043 & 3.425 & 9 & 3.43 & 0.036 & 2.866 & & & & \\
\hline Kidney & & & & & 265 & 1.18 & 0.034 & 2.526 & 300 & 1.19 & 0.013 & 0.767 \\
\hline Fetal liver & & & & & 48 & 1.53 & 0.042 & 3.529 & & & & \\
\hline Teratocarcinoma & & & & & & & & & 120 & 1.29 & 0.034 & 2.585 \\
\hline Aorta & & & & & & & & & 36 & 1.66 & 0.035 & 2.768 \\
\hline
\end{tabular}

To investigate cell types and tissues related to the differentially expressed gene profiles the DAVID software compared the lists of differentially expressed genes with normal physiologic expression of tissue-specific gene profiles. $\mathrm{N}$ is the number of differently expressed genes found in a tissue, FE is Fold Enrichment, Benjamini is the P-value after correction for multiple testing, and FDR is False Discovery rate.

is a collection of processes, making it difficult to recognize a central theme. Apart from apoptosis, these clusters describe normal cellular physiological processes taking also place in non-infected animals, e.g. during growth and development of the tissues and organs. Nevertheless, these processes also participate in the host reaction to infection with Salmonella.

Apart from small differences in the order of clusters the (e) and (et) categories of the DE group differ only in a few clusters from each other. A specific tyrosine phosphorylation was found in the (et) category but not in the (e) category of the DE group while the (e) category showed a protein domain WD cluster and a cell movement cluster, both not found in the (et) category. Finally, it should be noted that in none of the other categories (DE and IDD groups) a significant cluster with enrichment score of at least 2 could be found.

\section{Discussion}

The objective of this study was to determine the general chicken host response to Salmonella infection independent of age of the chicken host at time of bacterial challenge and independent of host response time postinfection, investigating various tissues and using chicken lines differing in susceptibility for Salmonella. The results highlight several biological mechanisms related to energy metabolism, apoptosis, specific protein domains indicating groups of involved proteins, and several cellular morphological structures where the affected processes are taking place. Overall, the reported meta-analysis approach showed successful integration of heterogeneous data sets of limited size by increasing statistical power. Using the results of this study for future biomarker analysis may provide in early diagnosis and warning of potentially hazardous food.

\section{Meta-analysis using data from different sources and different technologies}

A meta-analysis is performed using the original raw data from a number of individual experiments. Since the experiments may have different objectives and use different technologies, the experiments or data may not be directly comparable. In our study we compared data from four studies using: (1) genetically different chicken lines differing in Salmonella susceptibility), (2) different Salmonella serovars, (3) different sampling time points, (4) different sampled tissues, (5) different microarray types, and (6) different ages of bacterial challenge. 
Table 3 Clustering of gene lists using functional annotations

\begin{tabular}{|c|c|c|c|c|c|}
\hline $\begin{array}{l}\text { Enrichment } \\
\text { score (e) }\end{array}$ & $\begin{array}{l}\text { Enrichment } \\
\text { score (et) }\end{array}$ & $(e)^{1}$ & $(e t)^{1}$ & (t-deduced $)^{1}$ & Content Focus \\
\hline 10.09 & 10.46 & 1 & 1 & 1 & Lumen of organelles, specifically the nucleus \\
\hline 8.65 & 8.06 & 2 & 3 & 3 & $\begin{array}{l}\text { ATP / nucleotide binding; phosphorylation, (ser, thr) kinase, transferase, } \\
\text { S_TKC }\end{array}$ \\
\hline 8.15 & 8.14 & 3 & 2 & 2 & Mitochondrion (outer and inner membranes) \\
\hline 6.11 & 5.47 & 4 & 7 & $4-7$ & SH3 protein domain \\
\hline 5.94 & 6.30 & 5 & 5 & 5 & Mitochondrion \\
\hline 5.31 & 5.93 & 6 & 6 & 6 & Macromolecules, specifically protein catabolism, including UBL mechanism \\
\hline 5.00 & 6.42 & 7 & 4,24 & $4-7, \pm 20$ & Macromolecules / protein transport, especially import in nucleus / localization \\
\hline 4.83 & 4.28 & 8 & 8 & 8 & Non-membrane bound organelles and cytoskeleton \\
\hline 4.31 & 3.95 & 9 & 10 & $9-10$ & Cell cycle (process) \\
\hline 4.12 & 3.57 & 10 & 13 & \pm 15 & actin cytoskeleton (binding) \\
\hline 3.94 & 3.18 & 11 & 19 & $?$ & Protein folding / Chaperone protein \\
\hline 3.90 & 3.85 & 12 & 11 & 11 & Angiogenesis \\
\hline 3.73 & 3.69 & 13 & 12 & 12 & Ubl conjugation \\
\hline$X X X$ & 3.56 & $x$ & 14 & $<10$ & Tyrosine phosphorylation \\
\hline 3.68 & 3.44 & 14 & 15 & 15 & Endoplasmic reticulum \\
\hline 3.63 & $X X X$ & 15 & $x$ & $x$ & Ubiquitin / proteasome proteolysis \\
\hline 3.56 & 3.95 & 16 & 9 & $4-7$ & Transcription \\
\hline 3.52 & 3.22 & 17 & 17 & 17 & GTPase activity \\
\hline 3.03 & 3.22 & 18 & 18 & 18 & Muscle morphology \\
\hline 3.01 & $X X X$ & 19 & $x$ & $x$ & Intracellular vesicles \\
\hline 2.85 & 2.14 & 21 & 36 & $?$ & Cell-cell contacts \\
\hline 2.82 & 2.98 & 22 & 20 & 20 & Protein modification and metabolism, including proteolysis \\
\hline 2.75 & $X X X$ & 23 & $x$ & $x$ & Protein domain WD (repeat) \\
\hline 2.70 & 2.31 & 24 & 31 & $?$ & \\
\hline 2.70 & 2.68 & 25 & 23 & 23 & Apoptosis \\
\hline 2.69 & 2.72 & 26 & 22 & \pm 20 & RRM (RNA recognition motif) \\
\hline 2.64 & 2.27 & 27 & 32 & $?$ & Macromolecule complexes, especially protein complexes \\
\hline 2.45 & 2.07 & 31 & 38 & \pm 40 & Lysosome \\
\hline 2.40 & $X X X$ & 34 & $x$ & $x$ & Cell movement \\
\hline 2.37 & 2.21 & 35 & 33 & 33 & Mitochondrion / organelle outer membrane \\
\hline 2.21 & 1.67 & 36 & 52 & $?$ & Nucleotide binding via P-loop domain \\
\hline 2.08 & 1.94 & 38 & 41 & 41 & Nuclear pore / RNA transport \\
\hline 2.05 & 2.14 & 39 & 37 & 37 & Negative regulation of biosynthesis (nucleic acid, protein, macromolecules) \\
\hline 2.05 & 2.04 & 40 & 39 & 39 & Cellular response to diverse stimuli \\
\hline 1.03 & $2.2-1.55$ & 126 & $35+61$ & $<40$ & Muscle proteins, skeletal muscle morphology proteins \\
\hline
\end{tabular}

Clustering of the functional annotations of the gene lists of the differential expression (DE) group, i.e. the Number of Differentially Expressed genes (i.e. in the experiments and in the meta-analysis), and categories: (e): experiment - i.e. 4 studies, (t): time after infection - 14 different time point post-infection, and (et): overlap between the $(\mathrm{e})$ and $(\mathrm{t})$ groups. The t-values were deduced from the other groups because the number of data was too large for the DAVID software to analyze directly. 1: Number of the cluster within group.

Intuitively, it would be expected that these differences would affect the meta-analysis: (1) Genetically different lines of chicken, differing in Salmonella susceptibility, were expected to differ in reaction mechanism and/or reaction severity. (2) A pathogen specific host reaction was expected to different Salmonella serovars. (3) Sampling at different times post infection was suggested to show different temporal expression patterns related to the stage of infection. (4) Expression patterns are also expected to differ between different tissues or cell types. 
(5) Finally, different microarrays contained different sets of genes, so results from one study were expected to be missing from another study and vice versa. (6) The age of challenge of the birds would be expected to produce very different responses due to the poorly developed immune system of day old chicks compared to 3 week old birds. Despite of all these differences our meta-analysis indicated that the chicken lines react to Salmonella infection through comparable mechanisms irrespective of Salmonella serovar and tissue type, and therefore it may be concluded that we identified common mechanisms of the host response to the bacterial challenge. However, due to the different experimental ages of the animals used in the diverse studies, this conclusion may be hampered by the developmental differences of tissues and organs in the animals. It can be expected that at least part of the mechanisms found may relate to this. This could have been investigated only if control samples of all experimental ages in the individual datasets would have been available. But often these control samples are only available for the last experimental sampling age. Further experiments are needed to elucidate this point.

Although it is not certain, it can be expected that the results would have been more comprehensive if all experiments were performed under standard procedures. Similarly, the functional annotation analysis to elucidate potential biological mechanisms of the functional reaction of chicken to Salmonella infection would have been more robust.

\section{What does the functional annotation analysis teach us about the chicken host reaction to the infection with a Salmonella bacterium?}

The first indication that the chicken host reaction to Salmonella infection was similar between the diverse experiments was obtained from the similarities in the gene lists for the differently conducted experiment (e) and time $(\mathrm{t})$ categories, i.e. the (et) category. One unexpected finding was that the expression profiles related to several different cell types. Intestinal tissue is composed of many different cell types that could be indicated by a mixture of expression profiles. Furthermore, localized infection will change tissue expression profiles, which will be exacerbated by the influx of immune cells, which will further change the overall expression profile. However, the results indicated similarities to the expression profiles of several cell types including many unrelated tissues like liver and brain. The epithelium cell type of the intestine was the highest ranking tissue in all three analysis groups. Also platelet and muscle tissue, and perhaps colon expression profiles were recognizable - these cell types are also included in the intestinal tissue. Other cell types may also be on the list for several reasons. One reason may be that a cell type has a high turnover rate like epithelial cells in intestine tissue. In these cell types the general mechanism for cell division will be activated and therefore all these cell types appear on the list. Especially in developing young-age animals this may be expected. Finally, cell types and tissues may have been included in the list because we used the human physiological information instead of chicken physiological information for the DAVID software to create the list, e.g. lymph tissue may be inserted for that reason (although the chicken intestine contains a limited number of Peyer's patches as lymphoid tissues [38]).

The functional annotation is the result of the analysis of the DAVID software using the same gene lists to analyze several different databases containing biological function information. Due to the similarities within the gene lists the DE group (e), (t), and (et) categories showed similar functional annotations. Furthermore, the top category functional annotation "phosphoprotein" was also found in the differently expressed genes unique for both the experiments and the time (t) array after infection, and in the time-related genes found specifically in the meta-analysis (IDD-group). These results suggest two mechanisms: (1) the analysis is robust and indicated the same biological functionalities for all experiments despite the experimental differences, and (2) the metaanalysis adds new genes and data to the already existing data, but does indicate new biological mechanisms for the reaction of chicken hosts to the Salmonella infection. On the other hand, protein phosphorylation is an important regulatory mechanism for protein function in normal tissue and changed phosphoprotein content of the cell may have important physiological consequences for cellular metabolism (see below).

The clustering of biological functional annotations showed only in the lower part of the list differences between the DE (e) and DE ( $t$ ) category. While these differences themselves were statistically significant, the place on the list may suggest that the differences in the reaction of chicken to Salmonella are small. Alternatively, these differences point towards differences in the expression profiles related to time point after infection. However, due to the structure of the dataset these differences may also relate to deviations in the general chicken reaction mechanism caused by different chicken breed/lines, different tissues or different Salmonella serovars (jejunum vs. caecum; $S$. Enteritidis vs. $S$. Typhimurium).

While most clusters of differently expressed genes were similar in both the experiment (e) and the time (t) categories, some interesting differences were obtained. It should be noted that clusters found in one category but not in the other may be the result of real missing clusters or clusters failing to reach the enrichment score limit in one of the two categories. Three clusters were 
found in the differently expressed genes group experiment (e) category, but not in the time (t) category suggesting that these genes were not, or less regulated in time after infection and may be constitutively active during the chicken host reaction to Salmonella infection: (E1) Tyrosine phosphorylation, (E2) Protein domain WD (repeat), and (E3) cell movement. Two clusters were found in the differently expressed genes group time (t) category but not in the experiment (e) category, suggesting that these genes were especially regulated at different moments in time after infection of the chicken: (T1) ubiquitin / proteasome mediated proteolysis, and (T2) Intracellular vesicles.

\section{Tyrosine phosphorylation (E1)}

Phosphorylation activates or deactivates many proteins in cellular processes and protein phosphorylation in particular plays a significant role in a wide range of cellular processes [39-41]. Tyrosine phosphorylation is considered to be one of the key steps in signal transduction and regulation of enzymatic activity (for a review see [42]. The consequences of the difference between the (e)- and (t) categories (for tyrosine phosphorylation) may be important.

Both signal transduction and enzymatic activity may regulate a variety of important processes in the cell, including immune processes, cellular metabolism, and cell morphology, which may be related to the chicken host reaction to infection with Salmonella, e.g. via changes in the actin cytoskeleton $[43,44]$.

\section{Protein domain WD (E2)}

The WD40 repeat (also known as the WD or betatransducin repeat) is a short structural motif of approximately 40 amino acids, often terminating in a tryptophan-aspartic acid (W-D) dipeptide [45]. Several of these repeats are combined to form a type of protein domain called the WD domain. WD-containing proteins have 4 to 16 repeating units, all of which are thought to form a circularized beta-propeller structure [46,47]. WDrepeat proteins are a large protein family found in all eukaryotes and are implicated in a variety of functions ranging from signal transduction and transcription regulation to cell cycle control and apoptosis, which may be directly related to the chicken immune reaction to the Salmonella infection. All these specific functions were also found in other clusters. Thus, the difference between the (e) and (t) categories may induce modulations of the intensities of the processes described in several of the other clusters, thereby representing another mechanism for these proteins to modulate the chicken host response to Salmonella infection. Furthermore, the underlying common function of all WD-repeat proteins is coordinating multi-protein complex assemblies, where the repeating units serve as a rigid scaffold for protein interactions. The specificity of the proteins is determined by the sequences outside the repeats themselves. Several of the clusters relate to macromolecules which may be differently regulated between the $(\mathrm{e})$ and $(\mathrm{t})$ categories. A specific macromolecule includes the E3 ubiquitin ligase suggesting that also proteolysis is regulated [46,47].

\section{Cell movement (E3)}

Cell movement could relate to the influx of immune cells to the site of infection/ tissue. Also in non-infected tissues immune cells move through the tissue, but this process will be enhanced during infection. It may be suggested that regulation of this process may be one of the fundamental mechanisms of the cellular immune response of the chicken host.

\section{Ubiquitin / proteasome mediated proteolysis (T1)}

The destination of Ubiquitin tagged proteins is the proteasome for proteolysis. The ubiquination system functions in a wide variety of cellular processes, including the immune response and inflammation, antigen processing, apoptosis and cell cycle. Furthermore, the development and degeneration of several tissues is affected - probably via biogenesis of organelles such as ribosomes and modulation of cell surface receptors, ion channels, and the secretory pathway (for a review see [48]. The ubiquination system is responsive to stress and extracellular modulators such as Salmonella infection [49]. It is clear that the wide variety of cellular metabolic functions regulated by the ubiquitin / proteasome system may affect the chicken host response to Salmonella. Its regulation of expression especially at different time points after infection can modulate the response of the chicken host to Salmonella infection through a variety of mechanisms described in the other clusters.

\section{Intracellular vesicles (T2)}

Intracellular vesicles transport material - e.g. (macro) molecules - through the cell - either importing or exporting material, or transporting material to different cellular locations. They deliver molecules both for excretion or to lysosomes for degradation, and may import food components for energy and cellular components synthesis processes. Lotz et al. [50] described that the HSP90 protein is important for the regulation of intracellular vesicle transport. The HSP90 protein is a molecular chaperone regulating the folding and thereby the activity of macromolecules [51]. These functions can be found in several of the other clusters. Thus, the differential expression especially at different time points after infection can modulate the response of the chicken host to Salmonella infection during the cause of the 
infection through a variety of mechanisms described in the other clusters.

\section{Conclusions}

These results shed light on the important biological mechanisms that are active in the chicken gut cells during Salmonella infection - although part of the processes may relate to growth and development of the tissues and organs as discussed above. From our data we conclude that similar host mechanisms apply to $S$. Enteritidis and S. Typhimurium infection, and that similar biological mechanisms appear underlying the processes regulated during different times after infection.

\section{Additional files}

Additional file 1: The lists of genes with regulated expression in more than one or all experiments of the meta-analysis DE-group.

Additional file 2: Functional biological mechanisms of the differently expressed genes.

Additional file 3: Details of the biological functions per cluster.

\section{Abbreviations}

DE: The Number of Differentially Expressed genes; IDD: (Integration Driven Discoveries): the number of genes that were determined differentially expressed in the meta-analysis that were not identified in any of the individual studies alone (i.e. new differentially expressed genes); e: Experiment - i.e. 4 studies; t: Time after infection - 14 different time point post-infection; et: Overlap between the (e) and (t) groups; e-t: Genes unique in (e); t-e: Genes unique in (t).

\section{Acknowledgement}

The authors gratefully acknowledge financial participation from the European Community under the Sixth Framework Programme for Research,

Technological Development and Demonstration Activities, for the Integrated Project SABRE, Cutting Edge Genomics for Sustainable Animal Breeding, Contract no: FOOD-CT-2006-01625. The views expressed in this publication are the sole responsibility of the author(s) and do not necessarily reflect the views of the European Commission. Neither the European Commission nor any person acting on behalf of the Commission is responsible for the use, which might be made of the information. The information in this document is provided as is and no guarantee or warranty is given that the information is fit for any particular purpose. The user thereof uses the information at its sole risk and liability. Furthermore, additional finances were from the Kennisbasis (Knowledge Base) grants no KB-01-006, KB-04-004-006 and KB-04004-012 of the Dutch Ministry of Agriculture, Nature and Food security.

\section{Author details}

'Animal Breeding and Genetics Centre (ABGC), Wageningen UR Livestock Research, Animal Sciences Group, Wageningen University and Research Centre, P.O. Box 65, 8200 AB Lelystad, The Netherlands. ${ }^{2}$ Central Veterinary Institute - Department of Infectious Biology, Animal Sciences Group, Wageningen University and Research Centre, P.O. Box 65, 8200 AB Lelystad, The Netherlands. ${ }^{3}$ Institute for Animal Health, Genetics \& Genomics group, Compton, Berkshire, UK. ${ }^{4}$ INSERM UMR-S 945, Institut Fédératif de Recherches (IFR) 113, department of Immunité-Cancer-Infection, Hôpital Pitié-Salpêtrière, 83 Bld de l'Hôpital, Bâtiment CERVI, 75651 Paris, Cédex 13, France. ${ }^{5}$ INRA, AgroParisTech, UMR1313 Animal Genetics and Integrative Biology, F-78350 Jouy-en-Josas, France.

\section{Authors' contributions}

MtP: Coordinated the meta-analysis, participated in the design of the metaanalysis, participated in the analysis of the results of the meta-analysis, and drafted the manuscript. $1 \mathrm{H}$ : Participated in the design of the meta-analysis, performed the pre-processing of the data, participated in the meta-analysis, and helped in the analysis of the results of the meta-analysis. DS: Carried out the molecular genetic studies, analyzed the molecular results, participated in the design of the meta-analysis, participated in the meta-analysis, and helped in the analysis of the results of the meta-analysis. MS: Participated in the coordination the overall work in the project, participated in the coordination of the meta-analysis, participated in the design and analysis of the metaanalysis, and participated in drafting the manuscript. MF: Carried out the molecular genetic studies, analyzed the molecular results, and participated in drafting the manuscript. RZ: Carried out the animal studies, molecular genetic studies and immunologic reactions. M-LE: Carried out the animal studies, molecular genetic studies and immunologic reactions. AR:

Coordinated the overall work in the project, participated in the coordination of the meta-analysis, participated in the design and analysis of the metaanalysis, and participated in drafting the manuscript. All authors read and approved the final manuscript.

Received: 13 December 2011 Accepted: 24 April 2012

Published: 24 April 2012

\section{References}

1. Hofstad MS, John BH, Calnek BW, Reid WN, Yoder HW Jr: Diseases of Poultry. 8th edition. New Delhi, Ind: Panima Education Book Agency; 1992:65-123.

2. Kabir LSM: Avian Colibacillosis and Salmonellosis: A Closer Look at Epidemiology, Pathogenesis, Diagnosis, Control and Public Health Concerns. Int J Environ Res Public Health 2010, 7:89-114.

3. Wales AD, Davies RH: A critical review of Salmonella Typhimurium infection in laying Hens. Avian Pathol 2011, 40:429-436.

4. Chappell L, Kaiser P, Barrow P, Jones MA, Johnston C, Wigley P: The immunobiology of avian systemic salmonellosis. Vet Immunol Immunopathol 2009, 128:53-59.

5. Guard-Petter J: The chicken, the egg and Salmonella enteritidis. Environ Microbiol 2001, 3:421-430.

6. Parker MT: Enteric infections. In Topley and Wilson's principles of bacteriology, virology and immunity. Edited by Parker MT, Collier LH. London: Edward Arnold; 1990:424-446.

7. Barrow PA, Huggins MB, Lovell MA: Host Specificity of Salmonella Infection in Chickens and Mice Is Expressed In Vivo Primarily at the Level of the Reticuloendothelial System. Infect Immun 1994, 62:4602-4610.

8. Bumstead N, Barrow P: Resistance to Salmonella gallinarium, S. pullorium, and S. enteritidis in inbred lines of chicken. Avian Dis 1993, 37:189-193.

9. Van Hemert S, Hoekman AJW, Smits MA, Rebel JMJ: Gene expression responses to a Salmonella infection in the chicken intestine differ between lines. Vet Immunol Immunopathol 2006, 114:247-258.

10. Calenge F, Kaiser P, Vignal A, Beaumont C: Genetic control of resistance to salmonellosis and to Salmonella carrier-state in fowl: a review. Genet Selec Evol 2010, 42:11. doi:10.1186/1297-9686-42-11.

11. Redmond SB, Chuammitri P, Andreasen CB, Palić D, Lamont SJ: Proportion of circulating chicken heterophils and CXCLi2 expression in response to Salmonella enteritidis are affected by genetic line and immune modulating diet. Vet Immunol Immunopathol 2011, 140:323-328.

12. Hu J, Bumstead N, Barrow P, Sebastiani G, Olien L, Morgan K, Malo D: Resistance to Salmonellosis in the Chicken Is Linked to NRAMP1 and TNC. Genome Res 1997, 7:693-704.

13. Leveque G, Forgetta V, Morroll S, Smith AL, Bumstead N, Barrow P, LoredoOsti JC, Morgan K, Malo D: Allelic Variation in TLR4 Is Linked to Susceptibility to Salmonella enterica Serovar Typhimurium Infection in Chickens. Infect Immun 2003, 71:1116-1124.

14. Sadeyen J-R, Trotereau J, Velge P, Marly J, Beaumont C, Barrow PA, Bumstead N, Lalmanach A-C: Salmonella carrier state in chicken: comparison of expression of immune response genes between susceptible and resistant animals. Microbes Infect 2004, 6:1278-1286.

15. Fife M, Salmon N, Hocking P, Kaiser P: Fine mapping of the chicken salmonellosis resistance locus (SAL1). Anim Genet 2009, 40:871-877.

16. Pan Z, Fang Q, Geng S, Kang X, Cong Q, Jiao X: Analysis of immune-related gene expression in chicken peripheral blood mononuclear cells following Salmonella enterica serovar Enteritidis infection in vitro. Res Vet Sci 2012. 
17. Coble DJ, Redmond SB, Hale B, Lamont SJ: Distinct lines of chickens express different splenic cytokine profiles in response to Salmonella Enteritidis challenge. Poult Sci 2011, 90:1659-1663.

18. Galan JE, Cossart P: Host-pathogen interactions: a diversity of themes, a variety of molecular machines. Curr Opin Microbiol 2005, 8:1-3.

19. Mattoo S, Lee YM, Dixon JE: Interactions of bacterial effector proteins with host proteins. Curr Opin Immunol 2007, 19:392-401.

20. Te Pas MFW, van Hemert S, Hulsegge I, Rebel JMJ, Smits MA: A pathways analysis tool for analyzing microarray data of species with low physiological information. Adv Bioinf 2008. doi:10.1155/2008/719468. ID 719468.

21. Van Hemert S, Hoekman AJW, Smits MA, Rebel JMJ: Early host gene expression responses to a Salmonella infection in the intestine of chickens with different genetic background examined with cDNA and oligonucleotide microarrays. Comp Biochem Physiol D 2006, 1:292-299.

22. Schokker D, Hoekman AJW, Smits MA, Rebel JMJ: Gene expression patterns associated with chicken jejunal development. Dev Comp Immunol 2009, 33:1156-1164

23. Crhanova M, Hradecka H, Faldynova M, Matulova M, Havlickova H, Sisak F, Rychlik I: Immune response of chicken gut to natural colonization by gut microflora and to Salmonella enterica serovar enteritidis infection. Infect Immun 2011, 79:2755-2763.

24. Marot G, Foulley $J \mathrm{~L}$, Mayer $C D$, Jaffrézic F: Moderated effect size and Pvalue combinations for microarray meta-analyses. Bioinformatics 2009, 25:2692-2699.

25. Editorial: Meta-analysis and moderation. Nature Rev Drugs Disc 2010, 9:747.

26. Hedges LV, Olkin I: Statistical Methods for Meta-Analysis. NY, Academic Press: New York; 1985.

27. Stangl DK, Berry DA: Meta-Analysis in Medicine and Health Policy. New York, NY: Marcel Dekker; 2000.

28. Schokker D, Smits MA, Hoekman AJW, Parmentier HK, Rebel JMJ: Effects of Salmonella on spatial-temporal processes of jejunal development in chickens. Dev Comp Immunol 2010, 34:1090-1100.

29. Fife MS, Howel JS, Salmon N, Hocking PM, van Diemen PM, Jones MA Stevens MP, Kaiser P: Genome-wide SNP analysis identifies major QTL for Salmonella colonization in the chicken. Anim Genet 2010, 42:134-140.

30. Barrow PA, Bumstead N, Marston K, Lovell MA, Wigley P: Faecal shedding and intestinal colonization of Salmonella enterica in in-bred chickens: the effect of host genetic background. Epidemiol Infect 2003, 132:117-126.

31. Schokker D, Peters THF, Hoekman AJW, Rebel JMJ, Smits MA: Differences in the early response of hatchlings of different chicken breeding lines to Salmonella enterica serovar Enteritidis infection. Poultry Sci 2012, in press.

32. Smyth GK: Linear models and empirical bayes methods for assessing differential expression in microarray experiments. Stat Appl Genet Mol Biol 2004, 3:No. 1. Article 3.

33. Ritchie ME, Silver J, Oshlack A, Holmes M, Diyagama D, Holloway A, Smyth GK: A comparison of background correction methods for two-colour microarrays. Bioinformatics 2007, 23:2700-2707.

34. Huang DW, Sherman BT, Lempicki RA: Systematic and integrative analysis of large gene lists using DAVID Bioinformatics Resources. Nat Protoc 2009, 4:44-57.

35. Huang DW, Sherman BT, Lempicki RA: Bioinformatics enrichment tools: paths toward the comprehensive functional analysis of large gene lists. Nucl Acid Res 2009, 37:1-13.

36. http://david.abcc.ncifcrf.gov/.

37. Benjamini $Y$, Hochberg Y: Controlling the false discovery rate: A practical and powerful approach to multiple testing. J R Stat SoC B 1995, 57: 289-300.

38. Befus AD, Johnston N, Leslie GA, Bienenstock J: Gut-associated lymphoid tissue in the chicken. I. Morphology, ontogeny, and some functional characteristics of Peyer's patches. J Immunol 1980, 125:2626-2632.

39. Barford D, Das AK, Egloff MP: The structure and mechanism of protein phosphatases: insights into catalysis and regulation. Annu Rev Biophys Biomol Struct 1998, 27:133-164.

40. Van Weeren $P C$, de Bruyn $K M$, de Vries-Smits AM, van Lint J, Burgering BM: Essential role for protein kinase $B$ (PKB) in insulin-induced glycogen synthase kinase 3 inactivation. Characterization of dominant-negative mutant of PKB. J Biol Chem 1998, 273:13150-13156.

41. Blom N, Sicheritz-Pontén T, Gupta R, Gammeltoft S, Brunak S: Prediction of post-translational glycosylation and phosphorylation of proteins from the amino acid sequence. Proteomics 2004, 4:1633-1649.
42. Daniel JM, Reynolds AB: Tyrosine phosphorylation and cadherin/catenin function. Bioessays 1997, 19:883-891.

43. Galan JE, Zhou D: Striking a balance: Modulation of the actin cytoskeleton by Salmonella. Proc Natl Acad Sci USA 2000, 97:8754-8761.

44. Patel JC, Galan JE: Manipulation of the host actin cytoskeleton by Salmonella-all in the name of entry. Curr Opin Microbiol 2005, 8:10-15.

45. Neer EJ, Schmidt CJ, Nambudripad R, Smith TF: The ancient regulatoryprotein family of WD-repeat proteins. Nature 1994, 371:297-300.

46. Smith TF, Gaitatzes C, Saxena K, Neer EJ: The WD repeat: a common architecture for diverse functions. Trends Biochem Sci 1999, 24:181-185.

47. Li D, Roberts R: WD-repeat proteins: structure characteristics, biological function, and their involvement in human diseases. Cell Mol Life Sci 2001 58:2085-2097.

48. Pickart CM: Mechanisms underlying ubiquitination. Ann Rev Biochem 2001, 70:503-533.

49. Zhang Y, Higashide W, Dai S, Sherman DM, Zhou D: Recognition and ubiquitination of Salmonella type III effector SopA by a ubiquitin E3 ligase, HsRMA1. J Biol Chem 2005, 280:38682-38688.

50. Lotz GP, Brychzy A, Heinz S, Obermann WMJ: A novel HSP90 chaperone complex regulates intracellular vesicle transport. J Cell Sci 2008, 121: 717-723.

51. Csermely P, Schnaider T, Soti C, Prohászka Z, Nardai G: The 90-kDa molecular chaperone family: structure, function, and clinical applications. A comprehensive review. Pharmacol Ther 1998, 79:129-168.

doi:10.1186/1471-2164-13-146

Cite this article as: Pas et al:: Meta-analysis of Chicken - Salmonella infection experiments. BMC Genomics 2012 13:146.

\section{Submit your next manuscript to BioMed Central and take full advantage of:}

- Convenient online submission

- Thorough peer review

- No space constraints or color figure charges

- Immediate publication on acceptance

- Inclusion in PubMed, CAS, Scopus and Google Scholar

- Research which is freely available for redistribution 